\begin{tabular}{||l|l||}
\hline Citation & $\begin{array}{l}\text { Keivan Zavari, Hamid Khatibi, Vahid Johari Majd, Goele Pipeleers and Jan Swevers, 2011, } \\
\text { Fixed-order Robust Controller Design with Time-domain Constraints } \\
\text { American Control Conference (ACC), pp. 3698 - 3703 }\end{array}$ \\
\hline Archived version & $\begin{array}{l}\text { Author manuscript: the content is identical to the content of the published paper, but without } \\
\text { the final typesetting by the publisher }\end{array}$ \\
\hline Published version & article link on IEEE \\
\hline Publisher homepage & IEEE \\
\hline Author contact & $\begin{array}{l}\text { E-mail: keivan. zavari@kuleuven.be } \\
\text { Phone number: }+3216329261\end{array}$ \\
\hline IR & lirias, KULeuven link \\
\hline
\end{tabular}




\title{
Fixed-order Robust Controller Design with Time-domain Constraints
}

\author{
Keivan Zavari, Hamid Khatibi, Vahid Johari Majd*, Goele Pipeleers and Jan Swevers
}

\begin{abstract}
This paper deals with overshoot reduction in fixedorder controller design for linear systems subject to polytopic uncertainty. The basis of the developed synthesis method is a recent convex parameterization for fixed-order stabilizing controllers based on the polynomial approach. Two convex constraints are developed in order to decrease the overshoot of the closed-loop step response. First, based on the existing convex parameterization and peak-to-peak gain performance a criterion is developed to minimize the peak value of the step response. In the second method, Markov parameters of the system are used to achieve a step response with less overshoot. Simulation results illustrate the effectiveness of the developed methods.
\end{abstract}

\section{INTRODUCTION}

The computational complexity of modern controller design methods such as $\mathscr{H}_{\infty}$ and LQG reveals a watershed between full-order and reduced-order controllers. A full-order controller has the same order as the generalized plant (i.e. the plant plus frequency weights) and its design translates into a convex optimization problem, for which very efficient and reliable solvers exist [1], [2]. The reduced-order controller design, on the other hand, has not yet been reformulated as a convex optimization problem and consequently, it is much harder to solve. However, industry is often in preference of low-order controllers because of their simplicity, low cost, high reliability and low maintenance ([3], chap. 7).

The nonconvexity of the reduced-order controller design is inherited from the nonconvexity of the set of coefficients that yield a stable polynomial of degree larger than two. However, if the order of the controller is allowed to be higher than or equal to the generalized plant order, the problem can be reformulated as a convex problem by a change of variables. To overcome the nonconvexity of the reduced-order control problem, researcher resorted to convex but conservative stability conditions, yielding convex inner approximations of the set of stable polynomial coefficients. In [4] such a convex inner approximation is derived by transforming stability into strict positive realness (SPRness) with the help of the so-called central polynomial. SPRness of a transfer function invokes a convex constraint on the

K. Zavari, G. Pipeleers and J. Swevers are with PMA Division, Mechanical Engineering Department, Katholieke Universiteit Leuven, Belgium, keivan.zavari@mech.kuleuven.be

H. Khatibi and V. Johari Majd are with the School of Electrical and Computer Engineering, Tarbiat Modares University, Tehran, Iran

*Corresponding author, Associate Professor, Address: P.O. Box 14115143, Tehran, Iran, majd@modares.ac.ir Tel. Fax: +98 21 8288-3353 polynomial coefficients, and [4] transforms this constraint into a linear matrix inequality (LMI) based on polynomial positivity. With the help of the obtained inner approximation of the stability domain, [4] tackled the robust stabilization of linear systems subject to polytopic uncertainty by reducedorder controllers. In [5] a similar approach is taken, but the KYP lemma [6] is applied to transform the SPRness constraint into an LMI. In addition, the effect of the chosen central polynomial on the closed-loop poles is studied. In [7], the results of [4] are extended to the design of reduced-order controllers that optimize the worst-case $\mathscr{H}_{\infty}$ performance for polytopic systems. A convex parametrization of all stabilizing controllers is also given in [8], which can be readily used in the fixed-order controller design strategy of [5].

Concerning the control system's performance, many engineering applications demand a good step response, quantified in terms of overschoot, settling time, etc. Although these specifications can be addressed indirectly in an $\mathscr{H}_{2}$ or $\mathscr{H}_{\infty}$ design by choosing appropriate frequency weights, the selection of these weights is generally tedious and timeconsuming. Few control design approaches address timedomain specifications directly.

In [9], [10] Youla-Kučera controller parameterization and the polynomial approach are used to design a controller subject to time-domain constraints such as overshoot and input constraints. [11] also focuses on overshoot minimization in discrete-time systems and uses it for low-order controller design. Moreover, improving transient response is studied in [12] by a method called Characteristic Ratio Assignment (CRA). However none of these methods allows accounting both uncertainty and a fixed-order controller considering time-domain specifications, which is the subject of our paper.

This paper addresses the design of fixed-order robust controller subject to time-domain specifications. Hereto two additions to the fixed-order robust controller design procedure of [5] are proposed. The first approach is based on the peak-to-peak gain performance formulation provided in [13] and [14] for continuous- and discrete-time systems respectively. The second method shows that using system's Markov parameters can be employed to enhance the transient response of the system.

This paper considers polytopic uncertainty, which is one of the most general ways of representing structured uncertainties. It covers both the well-known interval and multi-model uncertainty. 
The remainder of this paper is organized as follows; Section II provides the preliminary background and the problem statement. In section III, the two methods for improving the time-domain response are developed and section IV] presents numerical validation of the approaches. Finally, in section $\mathrm{V}$ the concluding remarks are given.

\section{PROBLEM FORMULATION AND PRELIMINARIES}

In this section a brief outline of the design problem and mathematical preliminaries are provided.

\section{A. Problem Formulation}

Consider a SISO continuous-time or discrete-time system subject to parametric uncertainties residing in a polytope. That is, the set of potential plant models is given by

$$
G_{\delta}(\lambda)=\frac{b_{\delta}(\lambda)}{a_{\delta}(\lambda)}=\frac{\sum_{i=1}^{q} \delta_{i} b_{i}(\lambda)}{\sum_{i=1}^{q} \delta_{i} a_{i}(\lambda)}
$$

where $\lambda$ denotes the Laplace variable $s$ or shift operator $z$ and the vector $\delta \in \mathbb{R}^{q}$ comprises the uncertain parameters. It may vary in the following polytope $\boldsymbol{\delta}$ :

$$
\boldsymbol{\delta}=\left\{\delta \in \mathbb{R}^{q} \mid \delta_{i} \geq 0, \sum_{i=1}^{q} \delta_{i}=1\right\},
$$

while the q-"vertex" polynomials $a_{i}(\lambda)$ and $b_{i}(\lambda)$ are given by

$$
\begin{aligned}
& a_{i}(\lambda)=\lambda^{n}+a_{1, i} \lambda^{n-1}+\ldots+a_{n, i} \\
& b_{i}(\lambda)=b_{0, i} \lambda^{n}+b_{1, i} \lambda^{n-1}+\ldots+b_{n, i}
\end{aligned}
$$

Also consider a proper dynamic output feedback controller

$$
K(\lambda)=\frac{y(\lambda)}{x(\lambda)}=\frac{y_{0} \lambda^{m}+y_{1} \lambda^{m-1}+\ldots+y_{m}}{\lambda^{m}+x_{1} \lambda^{m-1}+\ldots+x_{m}}
$$

in the standard negative feedback configuration shown in Fig. 1. This yields the following closed-loop system $H_{\delta}(\lambda)$ from $r(t)$ to $z(t)$ :

$$
H_{\delta}(\lambda)=\frac{b_{\delta}(\lambda) y(\lambda)}{a_{\delta}(\lambda) x(\lambda)+b_{\delta}(\lambda) y(\lambda)}=\frac{b_{\delta}(\lambda) y(\lambda)}{c_{\delta}(\lambda)},
$$

where the denominator is the so-called characteristic polynomial of the closed-loop system and is indicated here by $c_{\delta}(\lambda)$ :

$$
c_{\delta}(\lambda)=\sum_{i=1}^{q} \delta_{i} c_{i}(\lambda)=\sum_{i=1}^{q} \delta_{i}\left(a_{i}(\lambda) x(\lambda)+b_{i}(\lambda) y(\lambda)\right)
$$

The aim of this paper is to derive a convex design procedure for reduced-order controllers $K(\delta)$ that (i) stabilize the closed-loop system; (ii) yield asymptotic tracking of a step input and (iii) minimize the step response overshoot; hereby explicitly accounting for the polytopic model uncertainty.

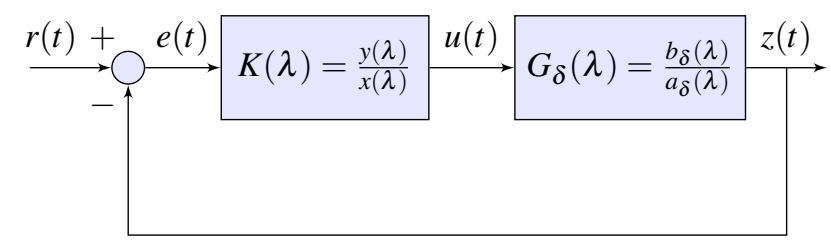

Fig. 1. Standard negative feedback configuration.

Robust closed-loop stability requires all the roots of $c_{\delta}(\lambda)$ to reside in the open left-half plane (continuous-time) or the unitary disc (discrete-time), for all $\delta \in \boldsymbol{\delta}$.

In addition, overshoot formulation invokes a nonconvex constraint on the controller parameters and in this paper it is translated to a convex one (Interested readers may want to see [9],[12],[15]).

\section{B. Convex Parameterization of fixed-order robust stabilizing controllers}

To overcome the nonconvexity of the fixed-order controller design problem, this paper adopts the approach of [5]. That is, the nonconvex coefficient set of stable polynomials is replaced by a convex inner approximation with the help of SPRness (KYP lemma) and a given stable polynomial, called the central polynomial.

The following lemma states the result of [5] for discretetime systems. It is straightforward to write the LMIs for continuous-time systems.

Lemma 1 ([5]): Consider the polytopic system $G_{\delta}(z)$ (1), the controller $K(z)$ (4) and a stable central polynomial $d(z)=z^{n+m}+d_{1} z^{n+m-1}+\ldots+d_{n+m}$. The closed-loop system is stable for all $\delta \in \boldsymbol{\delta}$ if the $q$ transfer functions $T_{i}(z)=\frac{c_{i}(z)}{d(z)}$ for $i=1, \ldots, q$ are SPR. The polynomial $c_{i}(z)$ corresponds to the characteristic polynomial on the $i$-th vertex, as is indicated in (6).

Let the coefficients of $K(z)$ be grouped in the vector $\chi$ :

$$
\chi=\left[\begin{array}{llllll}
x_{1} & \cdots & x_{m} & y_{0} & \cdots & y_{m}
\end{array}\right]^{T},
$$

and let $\left(A, B, C_{i}(\chi), D_{i}(\chi)\right)$ correspond to the controllable canonical state-space form of $T_{i}(z)$, then $K(z)$ robustly stabilizes the closed-loop system if there exists $P_{i}=P_{i}^{T}>0$ for $i=1, \ldots, q$ that satisfy:

$$
\left[\begin{array}{cc}
A^{T} P_{i} A-P_{i} & A^{T} P_{i} B-C_{i}(\chi)^{T} \\
B^{T} P_{i} A-C_{i}(\chi) & B^{T} P_{i} B-D_{i}(\chi)-D_{i}(\chi)^{T}
\end{array}\right]<0,
$$

Since the functions $C_{i}(\chi)$ and $D_{i}(\chi)$ are affine in $\chi$, considering $\chi$ as an additional optimization variable, does not compromise the linearity of the matrix inequalities (8).

This Lemma can be used to satisfy some performance measures as in [8] and is extended in [16] to satisfy $H_{\infty}$ performance with less conservatism than [8]. As stated above, 
one of the controller design specifications is to shape the closed-loop sensitivity function $S(\lambda)$ in frequency-domain, which invokes a constraint of the form $\left\|W_{1} S\right\|_{\infty}<\varepsilon$. In [16] it is shown that by using the relation between the bounded real lemma and the positive real lemma, the set of controllers that result in a closed-loop system with $\left\|W_{1}(\lambda) S_{\delta}(\lambda)\right\|_{\infty}<\varepsilon$ can be represented via LMIs as the Common Lyapunov Strictly Positive Realness (CL-SPRness) of the following transfer functions:

$$
\begin{aligned}
& \frac{c_{i}(\lambda)+\left(\varepsilon^{2}-1\right) a_{i}(\lambda) x(\lambda) W_{1}(\lambda)}{d(\lambda)}, \\
& \frac{c_{i}(\lambda)-\left(\varepsilon^{2}-1\right) a_{i}(\lambda) x(\lambda) W_{1}(\lambda)}{d(\lambda)}
\end{aligned}
$$

and this for all $i=1, \ldots, q$. Two equal-order SPR transfer functions are called CL-SPR if they satisfy the LMIs resulting from the KYP lemma with the same Lyapunov matrix $P$. The LMI representation of this theorem is straightforward due to the stated Lemma 1

\section{TIME-DOMAIN RESPONSE IMPROVEMENT}

This section presents two new methods for adding convex time-domain constraints to the optimization problem of the controller design. The first method is based on the peak-topeak gain performance formulation and is applicable to both discrete-time and continuous-time systems. The second one which is based on the definition of the time-domain signal, is simpler and yield better performance. The second approach uses the Markov parameters of the closed-loop system to convexify the objective function of the optimization problem.

\section{A. Minimizing the Peak-to-Peak Gain}

In order to reduce the peak amplitude of the output, the LMI constraints on the peak-to-peak gain for continuoustime and discrete-time systems are imposed. Unfortunately, these conditions are nonconvex when used for controller synthesis, similar to the matrix conditions for the $\mathscr{H}_{2}$ and $\mathscr{H}_{\infty}$ norm. In this part the peak-to-peak gain conditions are convexified to derive a controller resulting in less overshoot. The following lemma states the nonconvex LMI conditions for continuous-time and discrete-time systems ([13] and [14]).

Lemma 2: Suppose that $(A, B, C, D)$ is a state-space realization of the system $G(\lambda)$ with input signal $r(t)$ and output signal $z(t)$. Also assume that there exist $\mu, v \in \mathbb{R}$ and matrix
$P=P^{T}>0$ such that for continuous-time systems:

$$
\begin{aligned}
& \mu, v>0, \\
& {\left[\begin{array}{cc}
A^{T} P+P A+\mu P & P B \\
B^{T} P & -v I
\end{array}\right]<0,} \\
& {\left[\begin{array}{ccc}
\mu P & 0 & C^{T} \\
0 & (\gamma-v) I & D^{T} \\
C & D & \gamma I
\end{array}\right]>0}
\end{aligned}
$$

and for discrete-time systems:

$$
\begin{aligned}
& 0<\mu<1, v>0, \\
& {\left[\begin{array}{cc}
A^{T} P A-\mu P & A^{T} P B \\
B^{T} P A & -v I+B^{T} P B
\end{array}\right]<0,} \\
& {\left[\begin{array}{ccc}
(1-\mu) P & 0 & C^{T} \\
0 & \left(\gamma^{2}-v\right) I & D^{T} \\
C & D & I
\end{array}\right]>0,}
\end{aligned}
$$

then $\|G(\lambda)\|_{\text {peak }}<\gamma$, which implies that $\|z(t)\|_{\infty}<\gamma$ for any signal $r(t)$ with $\|r(t)\|_{\infty}<1$. 1 ${ }^{1}$ Moreover, all eigenvalues of $A$ are in the stability region (left-half plane or unit circle) or equivalently, $G(\lambda)$ is stable.

The LMIs of Lemma (2) are only linear in case of a fixed $\mu$. Hence, the best value of $\mu$ and consequently, the best bound of the provided formulation, is found by performing a line search over $\mu$.

It is also evident that the constraints of $(10 \mathrm{~b})$ and $(11 \mathrm{~b})$ are non-convex conditions for the controller synthesis because the controller parameters appear in the closed-loop statespace matrices. In [13] it is shown that for controllers of order at least equal to the plant order, these conditions can be convexified by a nonlinear change of controller variables. However, it remains nonconvex for the reducedorder controller design. Therefore we rely on the following theorem presents a sufficient condition to find a stabilizing fixed-order controller that satisfies the peak induced norm condition. This condition is convex such that the fixed-order design problem is again convex.

Theorem 1: Consider the closed-loop characteristic polynomial in (6), a stable central polynomial $d(\lambda)=\lambda^{n+m}+$ $d_{1} \lambda^{n+m-1}+\ldots+d_{n+m}$ and the vector $\chi$ defined in 77. In addition, let $\left(A, B, C_{i}(\chi), D_{i}(\chi)\right)$ denote the control canonical state-space form of $T_{i}(\lambda)=\frac{c_{i}(\lambda)}{d(\lambda)}$, while $\left(\mathscr{A}_{i}(\chi), \mathscr{B}_{i}(\chi), \mathscr{C}_{i}(\chi), \mathscr{D}_{i}(\chi)\right)$ indicates the control canonical state-space form of $H_{i}(\lambda)=\frac{b_{i}(\lambda) y(\lambda)}{c_{i}(\lambda)}$. Then, the closed-loop system is robustly stabilized and satisfies

$$
\left\|H_{\delta}(\lambda)\right\|_{\text {peak }}<\gamma, \quad \forall \delta \in \boldsymbol{\delta},
$$

\footnotetext{
${ }^{1}$ Considering $\quad x_{s}$ as the state variable of $G,\|G(\lambda)\|_{\text {peak }}=$ $\sup \left\{\|z(T)\|: x_{s}(0)=0, T \geq 0,\|r(t)\| \leq 1\right.$ for $\left.t \geq 0\right\}$
} 
if there exists $\mu_{i}, v_{i} \in \mathbb{R}$ and $P_{i}=P_{i}^{T}>0$, for $\forall i=1, \ldots, q$, such that

- for continuous-time systems:

$$
\begin{aligned}
& 0<\mu_{i}, 0<v_{i}, \\
& {\left[\begin{array}{ccc}
A^{T} P_{i}+P_{i} A+\mu_{i} P_{i} & P_{i} B-C_{i}(\chi)^{T} & P_{i} B \\
B^{T} P_{i}-C_{i}(\chi) & -D_{i}(\chi)-D_{i}(\chi)^{T} & 0 \\
B^{T} P_{i} & 0 & -v_{i} I
\end{array}\right]<0,} \\
& {\left[\begin{array}{ccc}
\mu_{i} P_{i} & 0 & \mathscr{C}_{i}(\chi)^{T} \\
0 & \left(\gamma-v_{i}\right) I & \mathscr{D}_{i}(\chi)^{T} \\
\mathscr{C}_{i}(\chi) & \mathscr{D}_{i}(\chi) & \gamma I
\end{array}\right]>0,}
\end{aligned}
$$

- for discrete-time systems:

$$
\begin{aligned}
& 0<\mu_{i}<1,0<v_{i}, \\
& {\left[\begin{array}{ccc}
A^{T} P_{i} A-\mu_{i} P_{i} & A^{T} P_{i} B-C_{i}(\chi)^{T} & A^{T} P_{i} B \\
B^{T} P_{i} A-C_{i}(\chi) & -D_{i}(\chi)-D_{i}(\chi)^{T}+B^{T} P_{i} B & 0 \\
B^{T} P_{i} A & 0 & -v_{i} I+B^{T} P_{i} B
\end{array}\right]<0} \\
& {\left[\begin{array}{ccc}
\left(1-\mu_{i}\right) P_{i} & 0 & \mathscr{C}_{i}(\chi)^{T} \\
0 & \left(\gamma^{2}-v_{i}\right) I & \mathscr{D}_{i}(\chi)^{T} \\
\mathscr{C}_{i}(\chi) & \mathscr{D}_{i}(\chi) & I
\end{array}\right]>0 .}
\end{aligned}
$$

Proof: The proof is given here for a continuous-time system, while the proof of the discrete-time result proceeds similarly. To prove our statement, it suffices to prove that

$$
\begin{aligned}
& {\left[\begin{array}{ccc}
A^{T} P+P A+\mu P & P B-C^{T} & P B \\
B^{T} P-C & -D-D^{T} & 0 \\
B^{T} P & 0 & -v I
\end{array}\right]<0 \Rightarrow} \\
& {\left[\begin{array}{cc}
\mathscr{A}^{T} P+P \mathscr{A}+\mu P & P \mathscr{B} \\
\mathscr{B}^{T} P & -v I
\end{array}\right]<0,}
\end{aligned}
$$

where $(A, B, C, D)$ denotes the controllable canonical statespace form of $T(s)=\frac{c(s)}{d(s)}$, while $(\mathscr{A}, \mathscr{B}, \mathscr{C}, \mathscr{D})$ equals the controllable canonical state-space form of $H(s)=\frac{b(s) y(s)}{c(s)}$. Applying the Schur complement to the left-hand side of (15) yields:

$$
\left[\begin{array}{cc}
A^{T} P+P A+\mu P+v^{-1} P B B^{T} P & P B-C^{T} \\
B^{T} P-C & -D-D^{T}
\end{array}\right]<0 .
$$

Similar to the proof of CL-SPRness of the transfer functions $T(s)$ and $T^{-1}(s)$, see e.g. [16], it can be shown that the inequality 16 holds for $(A, B, C, D)$ a state-space model for $T(s)$, if and only if it holds for a state-space model $(\tilde{A}, \tilde{B}, \tilde{C}, \tilde{D})$ of $T^{-1}(s)$ with the same Lyapunov matrix $P$. Since $T^{-1}(s)=\frac{d(s)}{c(s)}$ has the same denominator as $H(s)$, the matrices $\tilde{A}$ and $\tilde{B}$ may be chosen equal to $\mathscr{A}$ and $\mathscr{B}$, respectively. This way, the $(1,1)$ sub-block of the corresponding matrix inequality 16 yields:

$$
\begin{gathered}
\mathscr{A}^{T} P+P \mathscr{A}+\mu P+v^{-1} P \mathscr{B} \mathscr{B}^{T} P<0 \quad \Leftrightarrow \\
{\left[\begin{array}{cc}
\mathscr{A}^{T} P+P \mathscr{A}+\mu P & P \mathscr{B} \\
\mathscr{B}^{T} P & -v I
\end{array}\right]<0,}
\end{gathered}
$$

which completes the proof.

\section{B. Direct minimization of the output response using Markov parameters}

In this part, the step response of the discrete-time closedloop system is minimized through the use of Markov parameters. The time response of the $i$-th vertex of the polytopic closed-loop discrete-time system $H_{\delta}(\lambda)=\frac{b_{\delta}(\lambda) y(\lambda)}{c_{\delta}(\lambda)}$, see Eq. (5), is obtained by convolution as:

$$
z[k]=\sum_{l=0}^{k} g_{i}[k-l] r[l]=\sum_{l=0}^{k} g_{i}[l] r[k-l],
$$

where $g_{i}[k]$ is the system's impulse response from the reference input $r[k]$ to the output $z[k]$. By evaluating the state space equations at different time instances, and then repeatedly inserting the obtained expressions into each other to calculate $z[k]$ for all $k$, the system Markov parameters are characterized by:

$$
g_{i}[k]= \begin{cases}\mathscr{D}_{i} & k=0 \\ \mathscr{C}_{i} \mathscr{A}_{i}^{k-1} \mathscr{B}_{i} & k \geq 1,\end{cases}
$$

where $\left(\mathscr{A}_{i}(\chi), \mathscr{B}_{i}(\chi), \mathscr{C}_{i}(\chi), \mathscr{D}_{i}(\chi)\right)$ is the state space realization of the closed-loop transfer function.

At time-instance $k$, the difference between the closed-loop step response of the polytopic closed-loop system and the steady state value is bounded by $\gamma$ if and only if

$$
\begin{aligned}
& \mathscr{D}_{\delta}(\chi)+\mathscr{C}_{\delta}(\chi)\left(\sum_{k=0}^{N-1} \mathscr{A}_{\delta}(\chi)^{k}\right) \mathscr{B}_{\delta}(\chi)-1<\gamma, \\
& \forall N=0,1, \ldots, \quad \forall \boldsymbol{\delta} \in \boldsymbol{\delta} .
\end{aligned}
$$

This constraint is hard to impose due to the nonlinear dependency on $\delta$ and the nonlinear dependency on $\chi$ (nonconvex constraints), and hence it should be changed to a convex constraint for the optimization problem.

In [8] it is stated that the closed-loop poles can be assigned in a desired region by minimzing the norm

$$
\left\|\frac{c_{\delta}(\lambda)}{d(\lambda)}-1\right\|<\eta
$$

where $\|$.$\| can be any transfer funtion norm. It is also evident$ that in polytopic case, exact pole placement is not possible. However, the poles are assigned nearly around the desired ones if this norm is small enough and hence $H_{\delta}(\lambda)$ can be replaced with $T_{\delta}(\lambda)=\frac{b_{\delta}(\lambda) y(\lambda)}{d(\lambda)}$.

In order to convexify 20 using the mentioned replacement, the constraints required to bound the overshoot by $\gamma$ are approximated by:

$$
\begin{aligned}
& D_{i}(\chi)+C_{i}(\chi)\left(\sum_{k=0}^{N-1} A^{k}\right) B-1<\gamma, \\
& \forall N=0,1, \ldots, \quad \forall i=1, \ldots, q,
\end{aligned}
$$


where $\left(A, B, C_{\delta}(\chi), D_{\delta}(\chi)\right)$ denote the controllable canonical state-space form of $T_{\delta}(\lambda)$.

It is worth mentioning that since the constraint is linear in $\delta$ and $\chi$, it can be verified for the entire polytope by applying the constraint to each vertex. Summing up the constraint (21) at each time-instance gives $\gamma+\eta$ as the cost function to minimize and improve the time-domain response.

Remark 1: Constraint (21) still suffers from semiinfiniteness because of $N$ which is the length of the sequences considered in the constraint. It is evident that minimizing more sequences before the step response settles, leads to further improvement of the time response at the expense of computational complexity. Therefore, one has to determine a number $N$ based on the behavior of the closed-loop system.

\section{Simulation Results}

This section presents the numerical validation of the developed methods for robust fixed-order controller design. The following simulation results are obtained using MATLAB in combination with the SeDuMi solver [1], and the YALMIP interface [17].

First, consider the robust controller design problem taken from [8] which involves the following discrete-time system sampled at 1 second :

$$
G(z)=\frac{z+a}{z^{3}+b z^{2}+c z+d},
$$

where $a=0.2, b=-1, c=0.5, d=-0.1$ and all the parameters vary up to $\pm 7 \%$ from their nominal values. This uncertain system can be described by a polytope with $2^{4}=16$ vertices. Fig. 2 shows the bode plot of the systems at the vertices indicating that the uncertainty is large at low frequencies, which challenges robust control design.

To shape the sensitivity function the same weighting function as in [8] is used:

$$
W(z)=\frac{0.4902\left(z^{2}-1.0431 z+0.3263\right)}{z^{2}-1.282 z+0.282},
$$

which contains a pole at $z=1$, to enforce an integrator in the controller to achieve perfect reference step tracking in steady state. The inverse of $W(z)$ crosses the $-3 \mathrm{~dB}$ line from below at $2 \mathrm{rad} / \mathrm{sec}$.

The central polynomial $d(z)=\left(z^{2}-1.0432 z+0.3263\right)(z-$ $0.1)^{5}$, which has the desired closed-loop poles is also chosen the same as in [8] for a fourth order controller. The two desired closed-loop poles are related to the poles of a secondorder continuous-time system with a natural frequency of 0.7 $\mathrm{rad} / \mathrm{s}$ and a damping factor of 0.8 . The five auxiliary poles are selected much faster than the dominant poles and hence they are arbitrarily selected to be at $z=0.1$.

Three controllers of order four are compared. The first controller is designed using sensitivity shaping through equation (9) with $\varepsilon=0.7$ which corresponds to the design method

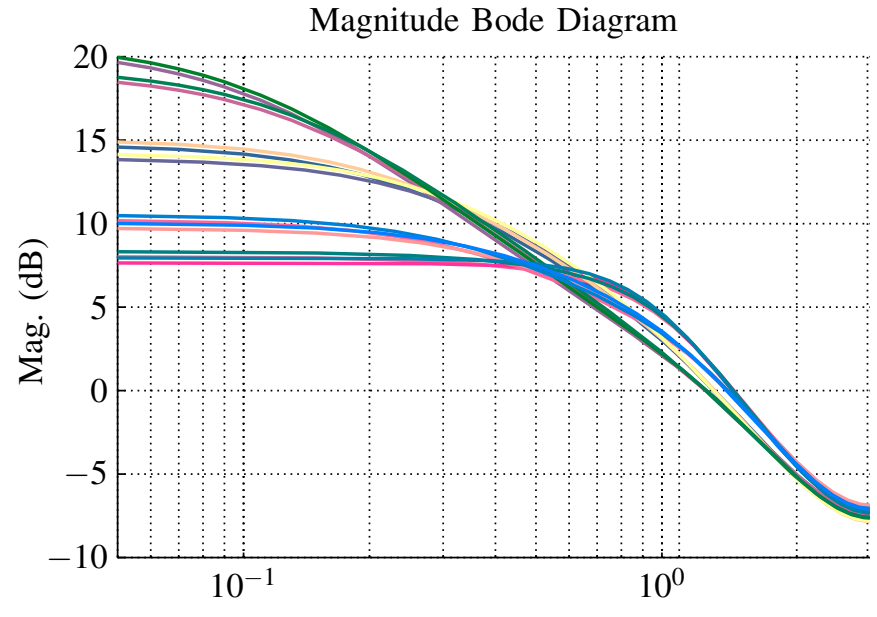

Freq. $(\mathrm{rad} / \mathrm{sec})$

Fig. 2. Magnitude Bode diagrams of all vertices of the polytopic system.

of [16]. The second and the third controllers are designed by in addition minimizing the peak-to-peak gain (method of section III-A and the output sequence using Markov Parameters (method of section III-B), respectively. Fig. 3 shows the closed-loop step response of the vertex system that yields the largest overshoot. Table (I) also summarizes the results of a fourth-order controllers for the polytopic system.

The results show that the second and the third controllers yield a significant reduction of the overshoot and their performance with respect to overshoot is comparable. The rise-time obtained with the third controller $(1.46 \mathrm{sec})$ is noticeably smaller than the rise time of the first controller $(2.75 \mathrm{sec})$ while the second method gives the rise-time of $17 \mathrm{sec}$. This can be explained by the conservativeness of the peak-to-peak gain method and also directly minimizing the error signal at all time instances for the Markov parameters method.

It is worth mentioning that the result in [8] is comparable to the shaping method of [16] shown in Fig. 3. because it only considers shaping the output sensitivity function and does not aim at improving the time-domain response.

\section{Conclusion}

In this paper convex constraints to enhance the timedomain response of polytopic systems in the robust fixedorder controller design problem are given. The peak-to-peak gain performance condition is convexified and used in fixedorder design procedure to minimize the overshoot of the step response. Based on the definition of Markov parameters a constraint is introduced to minimize the overshoot of the closed-loop step response in fixed-order controller design 
TABLE I

MAXIMUM STEP RESPONSE OVERSHOOT AND RISE-TIME OF THE CLOSED-LOOP POLYTOPIC SYSTEM OBTAINED BY THE PRESENTED METHODS.

\begin{tabular}{|l|l|l|}
\hline Optimization constraints & $\begin{array}{l}\text { Worst-case } \\
\text { overshoot }\end{array}$ & $\begin{array}{l}\text { Worst-case } \\
\text { rise-time (s) }\end{array}$ \\
\hline Sensitivity shaping & $39 \%$ & 2.75 \\
\hline $\begin{array}{l}\text { Sensitivity shaping and peak-to- } \\
\text { peak gain minimization }\end{array}$ & $21 \%$ & 17 \\
\hline $\begin{array}{l}\text { Sensitivity shaping and Markov pa- } \\
\text { rameters method }\end{array}$ & $19 \%$ & 1.66 \\
\hline
\end{tabular}

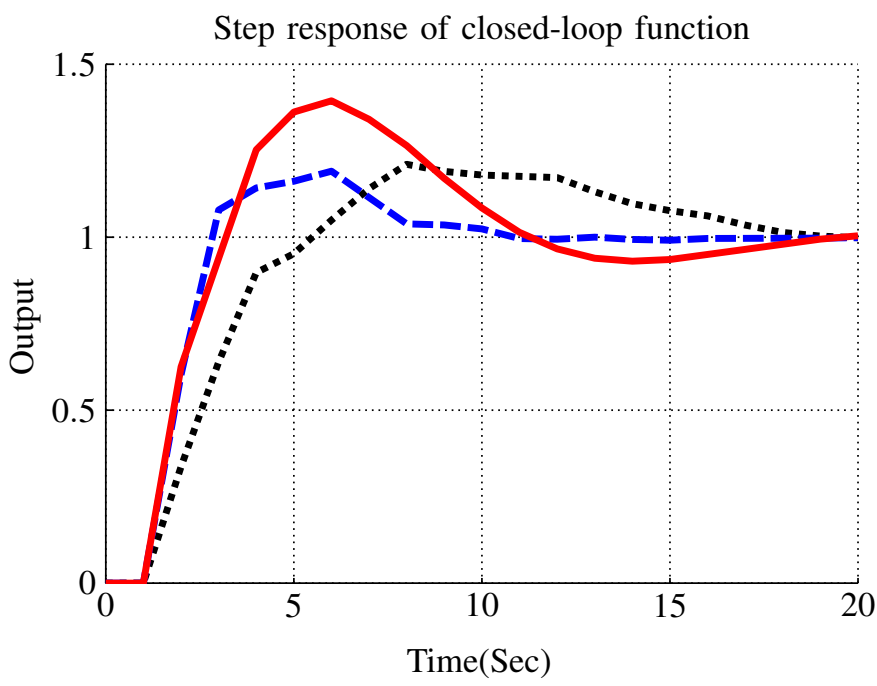

Fig. 3. Closed-loop step response of the worst vertex without time-domain constraints (solid), with peak-to-peak gain constraint (dotted), with Markov parameters method (dash).

for discrete-time systems. The effectiveness of the proposed methods is illustrated through numerical examples.

\section{ACKNOWLEDGMENTS}

Goele Pipeleers is a Postdoctoral Fellow of the Research Foundation - Flanders. This work was done in Tarbiat Modares University and benefits from projects G.0446.06, G.0422.08, and G.0377.09 of the Research Foundation Flanders (FWO-Vlaanderen), K.U.Leuven's Concerted Research Action GOA/05/10, K.U.Leuven's BOF EF/05/006 Optimization in Engineering Center (OPTEC), and the Belgian Program on Interuniversity Poles of Attraction IAP VI/4 DYSCO (Dynamic Systems, Control and Optimization) initiated by the Belgian State, Prime Minister's Office for Science, Technology and Culture. The scientific responsibility rests with its authors.

\section{REFERENCES}

[1] J. F. Sturm, "Using SeDuMi 1.02, a Matlab toolbox for optimization over symmetric cones," Optimization Methods and Software, vol. 11, pp. 625-653, 1999.

[2] K. C. Toh, M. J. Todd, and R. H. Tutuncu, "SDPT3: a MATLAB software package for semidefinite programming," Optimization Methods and Software, vol. 11, pp. 545-581, 1999.

[3] D. W. Gu, P. H. Petkov, and M. M. Konstantinov, Robust Control Design with MATLAB. Leipzig, Germany: Springer-Verlag, 2005.

[4] D. Henrion, M. Šebek, and V. Kučera, "Positive polynomials and robust stabilization with fixed-order controllers," IEEE Transactions on Automatic Control, vol. 48, no. 7, pp. 1178-1186, 2003.

[5] H. Khatibi, A. Karimi, and R. Longchamp, "Fixed-order controller design for systems with polytopic uncertainty using LMIs," IEEE Transactions on Automatic Control, vol. 53, no. 1, pp. 428-434, 2008.

[6] H. K. Khalil, Nonlinear Systems. New Jersey: Prentice Hall, 1996.

[7] F. Yang, M. Gani, and D. Henrion, "Fixed-order robust $H_{\infty}$ controller design with regional pole assignment," IEEE Transactions on Automatic Control, vol. 52, no. 10, pp. 1959-1963, 2007.

[8] A. Karimi, H. Khatibi, and R. Longchamp, "Robust control of polytopic systems by convex optimization," Automatica, vol. 43, no. 6, pp. 1395-1402, 2007.

[9] D. Henrion, S. Tarbouriech, and V. Kucera, "Control of linear systems subject to time-domain constraints with polynomial pole placement and LMIs," IEEE Transactions on Automatic Control, vol. 50, no. 9, pp. 1360-1364, 2005.

[10] — "Control of linear systems subject to input constraints: a polynomial approach," Automatica, vol. 37, pp. 597-604, 2001.

[11] O. N. Kiselev and B. Polyak, "Minimization of overshoot in linear discrete-time systems via low-order controllers," Automation and Remote Control, vol. 62, no. 4, pp. 597-606, 2001.

[12] Y. C. Kim, L. H. Keel, and S. P. Bhattacharyya, "Transient response control via characteristic ratio assignment," IEEE Transactions on Automatic Control, vol. 48, no. 12, pp. 2238-2244, December 2003.

[13] C. Scherer, P. Gahinet, and M. Chilali, "Multiobjective outputfeedback control via LMI optimization," IEEE Transactions on Automatic Control, vol. 42, no. 7, pp. 896-911, July 1997.

[14] J. M. Rieber, C. W. Scherer, , and F. Allgower, "Robust $L_{1}$ performance analysis for linear systems with parametric uncertainties," International Journal of Control, vol. 81, no. 5, pp. 851-864, 2008.

[15] K. L. Moore and S. Bhattacharyya, "A technique for choosing zero locations for minimal overshoot," IEEE Transactions on Automatic Control, vol. 35, no. 5, pp. 577-580, 1990.

[16] H. Khatibi and A. Karimi, "An alternative to the youla parameterization for $H_{\infty}$ controller design," in European Control Conference, Budapest, Hungary, August 2009.

[17] J. Löfberg, "Yalmip : A toolbox for modeling and optimization in MATLAB," in Proceedings of the CACSD Conference, Taipei, Taiwan, 2004. [Online]. Available: http://users.isy.liu.se/johanl/yalmip 